\title{
Mixing of Young Neutron Star Debris Into Supernova Envelopes
}

\author{
A. Noro, S. Miyaji \\ Grad. Sch. Science \& Technology, Chiba University, Chiba, Japan \\ K. Yamashita \\ University of Yamanashi, Kofu, Japan \\ T. Ogawa, M. Den \\ Communications Research Laboratory, Koganei, Japan
}

\begin{abstract}
We have simulated the 3D growth of a Rayleigh-Taylor instability in the expanding envelope of SN 1987A using the Adaptive Mesh Refinement Scheme utilizing a resolution of $4096^{-3}$. When the sinusoidal velocity perturbation amplitude is $10 \%$, heavy element tongues of the $\mathrm{He} / \mathrm{H}$ interface penetrate into the envelope with a high velocity.
\end{abstract}

\section{Introduction}

From observations of SN1987A, it is expected that radioactive elements around the core, i.e., the young neutron star, are well-mixed into the surrounding envelope. The debris of the young neutron star are assumed to be mixed by the Rayleigh-Taylor (RT) instability, and to result in clumps of heavy elements in the supernova remnant which are the source of $\gamma$-ray lines. By observing the widths of spectral lines of heavy elements in the infrared, Witteborn (1989) found that expansion velocities of heavy elements were $3000-4000 \mathrm{~km} \mathrm{~s}^{-1}$ in the expanding envelope of SN 1987A. Many numerical studies of the RT instability in the envelope have failed to reproduce observed high velocities (see e.g., Noro et al. 2002a). Using the Adaptive Mesh Refinement Scheme, we here show a simulation of the growth of the RT instability in 3D space until the clumps have saturated to their expanding velocity.

\section{Initial Conditions}

We used the spherical progenitor model of 14E1 of Shigeyama \& Nomoto (1990), shown as a dashed curve in the left panel of Figure 1. The total amount of mechanical energy converted into the shock wave by the supernova explosion is $1 \times 10^{51}$ ergs. We distributed the total energy into kinetic energy and thermal energy by the ratio of 1 to 4 initially (Den, Yoshida \& Yamada 1990) and followed the shock wave propagation with a spherical code. When the shock front had just 

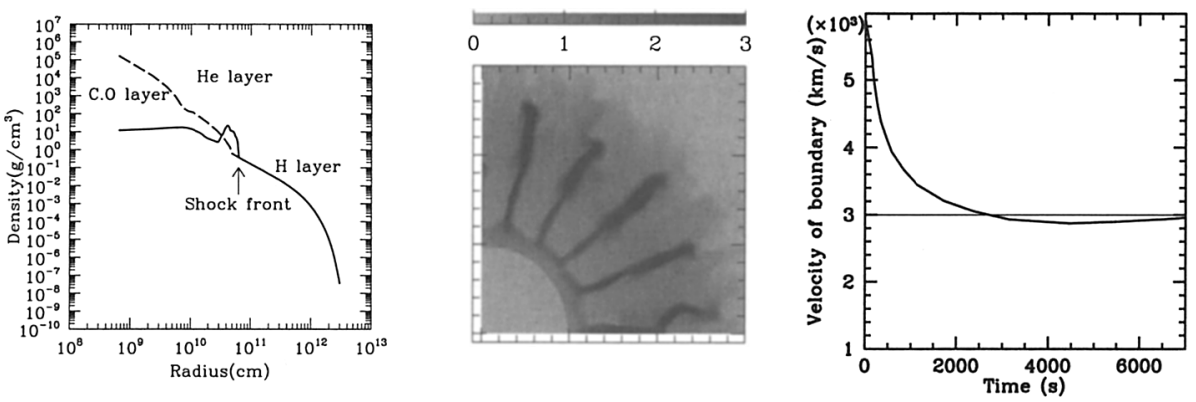

Figure 1. Left: Density vs radius at the start of our spherical simulatione (dashed curve) and at the start of our 3D simulation (solid curve). Center: The density profile in a slice of the equatorial plane at 7000 seconds. Right: The velocity of the $\mathrm{He} / \mathrm{H}$ interface against time.

passed the $\mathrm{He} / \mathrm{H}$ interface $(t=100 \mathrm{~s})$, we started our 3D simulation, beginning with the spherical simulation model (the solid curve in the left panel of Fig. 1), and providing a perturbation on the radial velocity inside the shock front of the form $\delta v_{r}(r, \theta, \phi)=v_{r} \epsilon \cos (m \theta) \sin (m \phi)$, where $v_{r}$ is the radial velocity, $\epsilon=0.1$ is the perturbation amplitude, and $m=20$ is the wavelength parameter. The size of the $3 \mathrm{D}$ simulation is $\left(2.25 \times 10^{12} \mathrm{~cm}\right)^{3}$ which is about half the size of the initial progenitor radius.

Such tongues keep their expanding velocity and would form heavy element clumps (Noro et al. 2002a). At the final stage of this simulation, the tongues have a velocity of $3000 \mathrm{~km} \mathrm{~s}^{-1}$, which successfully reproduces the velocity of the clumps observed.

\section{Results and Summary}

We followed the growth of the RT instability with a $10 \%$ perturbation until a time $t=7000 \mathrm{~s}$ after the explosion. The center panel of Figure 1 shows the density profile on the slice of equatorial plane at this time. The abscissa and ordinate are scaled by $\left(2.25 \times 10^{12} \mathrm{~cm}\right)^{3}$. The right panel of Figure 1 shows the velocity of the $\mathrm{He} / \mathrm{H}$ interface against time. After 2500 seconds, because of the balance between deceleration by surrounding gas and acceleration by the growth of RT instability, the velocity of the interface becomes stable and forms elongation tongues at the $\mathrm{He} / \mathrm{H}$ interface.

\section{References}

Den, M., Yoshida, T., \& Yamada, Y. 1990, Prog. Theor. Phys., 83, 723

Noro, A. et al. 2002a, Information Processing Society Journal Symposium Series, 4 , p. 9

Shigeyama, T., \& Nomoto, K. 1990, ApJ, 360, 242

Witteborn, F. C. et al. 1989, ApJ, 338, L9 